\title{
Findings Associated With a Novel Program Designed to Support Indigenous Faculty Members of U.S. Health Professions Schools
}

\author{
Patricia A. Carney, Cynthia Taylor, Allison Empey, Dove Spector, Amanda Bruegl, Erik Brodt
}

\section{A R T I C LE IN F O}

Keywords:

Faculty development

Indigenous faculty support

https://doi.org/10.32799/ijih.v16i1.33095
A UTHOR INFO

Patricia A. Carney, PhD, MS, Professor of Family Medicine, School of Medicine, Oregon Health \& Science University, Portland, Oregon, USA. E-mail: carneyp@ ohsu.edu. Lead and Corresponding Author, who oversaw drafting and critical revisions to the paper. Approved final version.

Cynthia Taylor, PhD, Research Associate, Department of Family Medicine, School of Medicine, Oregon Health \& Science University. Assisted with data analyses and drafting of manuscript. Approved final version.

Allison Empey, MD, Grande Ronde, Assistant Professor of Pediatrics, School of Medicine, Oregon Health \& Science University. Assisted with data interpretation, drafting manuscript and approved final version.

Dove Spector, Nez Perce, Research Assistant, Department of Family Medicine, School of Medicine, Oregon Health \& Science University. Assisted with drafting manuscript and approved final version.

Amanda Bruegl, MD, MS, Oneida, Stockbridge-Munsee, Assistant Professor of Obstetrics and Gynecology, School of Medicine, Oregon Health \& Science University. Assisted with drafting manuscript and approved final version. 
Erik Brodt, MD, Ojibwe, Associate Professor of Family Medicine, School of Medicine, Oregon Health \& Science University. Assisted with data interpretation, drafting manuscript and approved final version.

\section{Acknowledgements}

This work was supported by the Northwest Native American Center of Excellence (NNACoE), funded by the Health Resources and Services Administration, Bureau of Health Workforce under grant number D34HP31026 for NNACoE.

\section{Introduction}

It is well known that a diverse workforce in the health professions will improve health equity (Cohen et al., 2002); however, diversity at the faculty level continues to lag behind diversity at the student level (Page et al., 2011). Challenges have been reported regarding the academic success of underrepresented minority (URM) faculty members. A study by Fang et al. (2000) found that White faculty had significantly more first-authored and total peer-reviewed publications than minority faculty groups. After adjustments for relevant covariates, this study found that the odds of holding senior rank were significantly lower for Black faculty than for White faculty $(0.33 ; 95 \%$ confidence interval [0.17, 0.63]). At least one other study on this topic found that URMs are more likely than Whites to have fewer and inadequate educational opportunities that affect their preparation for certain careers and can undermine their ability to gain qualifications for both training to attain advanced degrees and faculty positions (Price et al., 2005).

Academic institutions often lack culturally relevant resources, such as mentors that could bolster retention among URM faculty (Fang et al., 2000). One study found that $48 \%$ of URM faculty perceived racial/ethnic bias in their academic environment and had lower levels of career satisfaction compared to $26 \%$ of their non-URM counterparts (Peterson et al., 2004). Recent studies on programs designed to support URM faculty are lacking, which is especially true for Indigenous people who lag behind the general population on many socioeconomic and health indices, such as educational attainment, morbidity, and mortality (Bowman, 2008; Kunitz, 2008; Marcinko, 2016; Northwest Portland Area Indian Health Board, 2014; U.S. Census Bureau, 2010). Expanding the numbers of successful Indigenous health faculty could improve delivery of culturally appropriate/responsive health care for Indigenous people (Bowman, 2008; Marcinko, 2016).

Of great concern is that the number of American Indian and Alaska Native (AIAN) U.S. medical school applicants declined by 32\% between 1980 and 2013 (Association of American Medical Colleges, 2017). Moreover, this negative trend is accelerating with a $70 \%$ decline in AIAN applicants and 63\% decline in AIAN matriculants to U.S. medical schools between 1996 and 2016 (Association of American Medical Colleges, 2017). Similarly, Native Hawaiian and other Pacific Islander (NHPI) matriculation to medical school has decreased by $46 \%$ over the past 4 years (Association of American Medical Colleges, 2017). An inadequate pool of AIANs and NHPIs matriculating into medical school directly leads to an inadequate pool of Indigenous medical school faculty. Our own prior work has shown that U.S. Indigenous faculty members, 
defined as AIAN and NHPI, represented the smallest percentage of U.S. allopathic medical school faculty at $0.11 \%$ and $0.18 \%$ respectively (Brodt et al., 2018), and that Indigenous faculty represented the lowest percentage of full professor faculty at 5.2\% in 2016 for AIAN faculty and a decline from $4.6 \%$ to $1.6 \%$ between 2014 and 2016 for NHPI faculty ( $p<.001$; Brodt et al., 2018).

Studies that both describe and assess the effectiveness of strategies to support Indigenous faculty members are lacking. To address this gap in existing literature, we report on findings from two cohorts of AIANs and NHPIs who participated in an Indigenous Faculty Forum (IFF) program designed to support Indigenous faculty as part of the Northwest Native American Center of Excellence, based in Portland, Oregon, USA.

\section{Methods}

\section{Overview}

The Northwest Native American Center of Excellence, co-led by the principal investigator (author EB) and deputy director (author AE), represents a partnership between: (a) Oregon Health \& Science University, the only medical school in the state of Oregon, (b) Portland State University, and (c) the Northwest Portland Area Indian Health Board, a nonprofit organization representing the 43 federally recognized tribes of the Pacific Northwest. The educational programs of the Northwest Native American Center of Excellence are diverse and are described in detail elsewhere (Brodt et al., 2019). Briefly, these include Wy'east, a 9-month (September through May) post-baccalaureate pathway designed specifically to prepare AIANs to earn acceptance to and excel in medical school; Tribal Health Scholars, a 14-week clinical shadowing experience for AIAN high school students that exposes them to various health professional careers in their community's health clinic; and the IFF, a program designed to promote the advancement of AIANs in academic medicine. Findings from the IFF are the focus of this paper.

\section{Ethics}

The Northwest Native American Center of Excellence includes a team of experts in educational research and program evaluation. This team oversees all learner assessments and program evaluation in accordance with Oregon Health \& Science University and Northwest Portland Area Indian Health Board's Institutional Review Boards (IRB No. 17588).

\section{Indigenous Faculty Forum}

Because AIAN faculty play an important role in training the future health care workforce, it is vital to support their professional development and academic success. The objective of IFF is to create an AIAN faculty development conference designed to enhance the success of current and future AIAN academic health faculty. During the sessions, we provide instruction in key professional competencies pertaining to academic advancement, address unique cultural considerations for Indigenous faculty, and foster networking and ongoing career support. Ideally, the networking that occurs will create an informal cohort effect for ongoing peer support and 
further opportunities for collaboration. The educational design of IFF addresses the unique challenges experienced by Indigenous faculty, such as limited information on academic career paths, isolation, challenges in balancing Indigenous and academic cultures, and lack of role models and mentors (Sánchez et al., 2016). Additionally, Indigenous faculty, like other URM faculty, experience the "minority tax," being burdened with increased institutional service commitments to help with diversity efforts, racism, and inequities in advancement and promotion (Rodriguez et al., 2015).

The Northwest Native American Center of Excellence conducts the day-long IFF session annually with the first session held in November 2017 and hosted in Portland, Oregon. This first event was considered a pilot year and nine participants attended, which represented 9 of the 18 (50\%) identified AIAN academic faculty who practice on the West Coast of the continental U.S. In the second year (July 2018), the IFF was hosted in Hilo, Hawaii, as part of the Pacific Region Indigenous Doctors Congress, with 53 participants. Attendees at the second session were Indigenous physicians and professors from U.S. and Pacific Rim medical schools, representing a broad assortment of medical specialties and departments. The IFF session began with cultural protocols and took place in a traditional Hawaiian 'ohana (family) style of building, which created an open, comfortable atmosphere intended to facilitate open and natural discussions. The agenda included lectures, small group sessions, and expert panels. Topics included historical trauma, racism, and microaggression in academia, strategic career planning, maintaining one's own cultural identity within the larger institutional culture, mentorship, and building bridges with tribal clinics and communities.

Participants for the pilot IFF cohort were recruited through outreach to West Coast medical schools, the Association of American Indian Physicians, and word of mouth by other AIAN academic faculty. The most successful means of identifying participants for the pilot IFF was through word of mouth by AIAN academic faculty. Participants for the second IFF were recruited from the AIAN academic faculty identified for the pilot cohort and through the Pacific Region Indigenous Doctors Congress informational materials and registration process. The second IFF was held as a pre-conference to the Pacific Region conference and increased the pool of potential Indigenous faculty participants.

\section{Data Collection and Analyses}

The evaluation design for this study was quasi-experimental mixed methods. Specific evaluation activities included a post-session IFF program evaluation, a focus group held immediately after the IFF session, and a 12-month follow-up survey designed to assess how participants' careers were developing. The post-session program evaluation determined participants' demographic and practice information, including gender identity (including twospirit/nonbinary), age, faculty rank, Indigenous affiliation, number of years in practice, number of years as a faculty member, and primary specialty. It also used the 22-item Diversity Engagement Survey (DES; Person et al., 2015), which consists of eight factors that connect engagement theory to diversity and inclusion constructs. Research using this tool has shown that black, Hispanic/Latino, female, and LGBTQ (lesbian, gay, bisexual, transgender, queer) 
respondents reported lower degrees of engagement than their racial majority counterparts (Person et al., 2015).

The eight factors of the DES are as follows:

1. Common Purpose. Individuals experience a connection to the mission, vision, and values of the organization.

2. Access to Opportunity. Individuals perceive that they are able to find and utilize support for their professional development and advancement.

3. Equitable Reward and Recognition. Individuals perceive the organization as having equitable compensation practices and nonfinancial incentives.

4. Cultural Competence. Individuals believe the institution has the capacity to make creative use of its diverse workforce in a way that meets business goals and enhances performance.

5. Trust. Individuals have confidence that the policies, practices, and procedures of the organization will allow them to bring their best and full selves to work.

6. Sense of Belonging. Individuals experience their social group identity as being connected with and accepted in the organization.

7. Appreciation of Individual Attributes. Individuals perceive they are valued and can successfully navigate the organizational structure in their expressed group identity.

8. Respect. Individuals experience a culture of civility and positive regard for diverse perspectives and ways of knowing.

All 22 items are worded positively and are scored using a five-point Likert scale of $1=$ strongly disagree to $5=$ strongly agree. Survey data were collected immediately following the IFF day-long sessions, and web-based follow-up surveys were administered 1 year after the respective IFF sessions were held, with three reminders to complete the survey before it closed.

The focus groups occurred immediately after the day-long sessions and were facilitated by trained faculty/staff. Focus group questions included the following:

- How has your academic career gone so far?

- What has made a difference in fostering career success?

- What are you most proud of in terms of academic accomplishments?

- What makes an academic career challenging?

- How has your culture fostered or hindered your success as a professional?

- How does your culture inform your experience as an academic faculty member?

- What types of professional development help do you need?

Two observers took field notes during the session, and these notes were integrated into a composite document for analyses. The 1-year follow-up survey included questions about changes in faculty rank, changes in roles for teaching activities, changes in tribal community engagement, and diversity activities. In addition, the DES was re-administered to see if any notable changes were occurring at their institutions over time. 
Quantitative analyses included running descriptive statistics, including means, standard deviations, and frequencies. Comparisons of participant characteristics in Cohort 1 and Cohort 2 were done two ways. First all participants were included and analysis of variance (ANOVA) was used to assess independent samples for differences; then surveys were linked to the same respondents in the post-session and 1-year follow-up surveys using Wilcoxon signed rank test. Though the values changed slightly in the linked versus independent samples, the statistical assessment did not differ; thus, we are presenting the independent samples findings here.

Qualitative analyses included a content analysis (Mayring, 2000) to identify responses mentioned by more than three participants.

\section{Relationship}

Dr. Brodt is the founder and president of We Are Healers, a nonprofit organization involved in this project. If you would like more information, please contact the Oregon Health \& Science University Research Integrity Office at 503-494-7887 or coir@ohsu.edu.

\section{Results}

Nine AIANs participated in the first IFF session (2017) and 100\% completed the postsession survey and participated in the focus group. Eight of them completed the 1-year follow-up survey for an $88.9 \%$ response rate. Fifty-three Indigenous faculty participated in the second IFF session (2018), and of these, 29 completed the post-IFF survey (54.7\%) and 12 participated in the focus group (22.6\%). Thirty completed the 1-year follow-up survey (56.6\%). Participants in both IFF sessions were predominantly female (Table 1) and were most often aged 35-44. They were most often assistant professors and had been in clinical practice for a mean of 14-16.3 years and faculty members for between 7.8 and 8.8 years on average (Table 1). They represented a diverse group of specialties in the health professions (Table 2).

\section{Table 1}

Post-Session and 1-Year Follow-Up Demographic Information for Indigenous Faculty Forum Cohorts 1 and 2

\begin{tabular}{|l|c|c|c|c|c|c|}
\hline \multirow{2}{*}{ Characteristic } & \multicolumn{3}{|c|}{ Post-session } & \multicolumn{3}{c|}{ 1-Year follow-up } \\
\cline { 2 - 7 } & $\begin{array}{c}\text { Cohort 1 } \\
(\boldsymbol{n}=\mathbf{9})\end{array}$ & $\begin{array}{c}\text { Cohort 2 } \\
(\boldsymbol{n = 2 9 )}\end{array}$ & $\begin{array}{c}\text { Cohorts } \\
\text { combined } \\
(\boldsymbol{n}=\mathbf{3 8})\end{array}$ & $\begin{array}{c}\text { Cohort 1 } \\
(\boldsymbol{n}=\mathbf{8})\end{array}$ & $\begin{array}{c}\text { Cohort 2 } \\
(\boldsymbol{n}=\mathbf{3 0})\end{array}$ & $\begin{array}{c}\text { Cohorts } \\
\text { combined } \\
(\boldsymbol{n}=\mathbf{3 8})\end{array}$ \\
\hline Gender & $\boldsymbol{n}(\boldsymbol{\%})$ & $\boldsymbol{n}(\boldsymbol{\%})$ & $\boldsymbol{n}(\boldsymbol{\%})$ & $\boldsymbol{n}(\boldsymbol{\%})$ & $\boldsymbol{n}(\boldsymbol{\%})$ & $\boldsymbol{n}(\boldsymbol{\%})$ \\
\hline Female & $8(88.9)$ & $23(79.3)$ & $31(81.6)$ & $7(87.5)$ & $20(66.7)$ & $27(71.1)$ \\
\hline Male & $1(11.1)$ & $6(20.7)$ & $7(18.4)$ & $1(12.5)$ & $10(33.3)$ & $11(28.9)$ \\
\hline
\end{tabular}


Table 1 con't

\begin{tabular}{|c|c|c|c|c|c|c|}
\hline \multirow[b]{2}{*}{ Characteristic } & \multicolumn{3}{|c|}{ Post-session } & \multicolumn{3}{|c|}{ 1-Year follow-up } \\
\hline & $\begin{array}{c}\text { Cohort } 1 \\
(n=9)\end{array}$ & $\begin{array}{l}\text { Cohort } 2 \\
(n=29)\end{array}$ & $\begin{array}{l}\text { Cohorts } \\
\text { combined } \\
(n=38)\end{array}$ & $\begin{array}{c}\text { Cohort } 1 \\
(n=8)\end{array}$ & $\begin{array}{c}\text { Cohort } 2 \\
(n=30)\end{array}$ & $\begin{array}{c}\text { Cohorts } \\
\text { combined } \\
(n=38)\end{array}$ \\
\hline Age & $n(\%)$ & $n(\%)$ & $n(\%)$ & $n(\%)$ & $n(\%)$ & $n(\%)$ \\
\hline $25-34$ & 0 & $5(17.2)$ & $5(13.2)$ & 0 & $3(10.0)$ & $3(7.9)$ \\
\hline $35-44$ & $6(66.7)$ & $9(31.0)$ & $15(39.5)$ & $5(62.5)$ & $8(26.7)$ & $13(34.2)$ \\
\hline $45-54$ & $2(22.2)$ & $6(20.7)$ & $8(21.1)$ & $2(25.0)$ & $8(26.7)$ & $10(26.3)$ \\
\hline Older than 54 & $1(11.1)$ & $7(24.1)$ & $8(21.1)$ & $1(12.5)$ & $11(36.7)$ & $12(31.6)$ \\
\hline Missing & 0 & $2(6.9)$ & $2(5.3)$ & 0 & 0 & 0 \\
\hline Faculty rank & $n(\%)$ & $n(\%)$ & $n(\%)$ & $n(\%)$ & $n(\%)$ & $n(\%)$ \\
\hline Instructor & $1(11.1)$ & $5(17.2)$ & $6(15.8)$ & 0 & $2(6.7)$ & $2(5.3)$ \\
\hline Assistant professor & $3(33.3)$ & $11(37.9)$ & $14(36.8)$ & $4(50.0)$ & $9(30.0)$ & $13(34.2)$ \\
\hline Associate professor & $3(33.3)$ & $4(13.8)$ & $7(18.4)$ & $3(37.5)$ & $5(16.7)$ & $8(21.1)$ \\
\hline Professor & 0 & $3(10.3)$ & $3(7.9)$ & 0 & $2(6.7)$ & $2(5.3)$ \\
\hline Other $^{\mathrm{a}}$ & $2(22.2)$ & $6(20.7)$ & $8(21.1)$ & $1(12.5)$ & $1(3.3)$ & $2(5.3)$ \\
\hline Missing & 0 & 0 & 0 & 0 & $11(36.7)$ & $11(28.9)$ \\
\hline $\begin{array}{l}\text { Indigenous } \\
\text { affiliation }\end{array}$ & $n(\%)$ & $n(\%)$ & $n(\%)$ & $n(\%)$ & $n(\%)$ & $n(\%)$ \\
\hline American Indian & $9(100.0)$ & $7(24.1)$ & $16(42.1)$ & $8(100.0)$ & $10(33.3)$ & $18(47.4)$ \\
\hline $\begin{array}{l}\text { First Nations } \\
\text { (Canada) }\end{array}$ & 0 & $5(17.2)$ & $5(13.2)$ & 0 & $4(13.3)$ & $4(10.5)$ \\
\hline Native Hawaiian & 0 & $2(6.9)$ & $2(5.3)$ & 0 & $4(13.3)$ & $4(10.5)$ \\
\hline $\begin{array}{l}\text { Other Indigenous } \\
\text { people }\end{array}$ & 0 & $13(44.8)$ & $13(34.2)$ & 0 & $9(30.0)$ & $9(23.7)$ \\
\hline Non-Indigenous & 0 & $1(3.4)$ & $1(2.6)$ & 0 & $2(6.7)$ & $2(5.3)$ \\
\hline Missing & 0 & $1(3.4)$ & $1(2.6)$ & 0 & $1(3.3)$ & $1(2.6)$ \\
\hline Years in practice ${ }^{\mathrm{b}}$ & Mean $(S D)$ & Mean $(S D)$ & & & & \\
\hline Number of years & $14(9.4)$ & $16.3(10.4)$ & & & & \\
\hline Range & $5-34$ & $1-26$ & & & & \\
\hline $\begin{array}{l}\text { Years as faculty } \\
\text { member }\end{array}$ & Mean $(S D)$ & Mean $(S D)$ & & & & \\
\hline Number of years & $8.8(6.8)$ & $7.8(8.9)$ & & & & \\
\hline Range & $1-26$ & $0.25-22$ & & & & \\
\hline
\end{tabular}

a Post-session for Cohort 1, "Other" included 1 fellow and 1 tribal liaison. For Cohort 2, "Other" included three lecturers, and one each of the following: clinician, research associate/PhD student, and staff. ${ }^{b}$ Years of practice was calculated by subtracting the year of graduation from a medical or $\mathrm{PhD}$ program from the current year (2018). 
Table 2

Primary Specialty According to Cohort at Post-Session and 1-Year Follow-Up Surveys

\begin{tabular}{|l|c|c|c|c|}
\hline \multirow{2}{*}{ Primary specialty } & \multicolumn{2}{|c|}{ Post-session } & \multicolumn{2}{c|}{ 1-Year follow-up } \\
\cline { 2 - 5 } & $\begin{array}{c}\text { Cohort 1 } \\
(\boldsymbol{n = 9 )}\end{array}$ & $\begin{array}{c}\text { Cohort 2 } \\
(\boldsymbol{n = 2 9 )}\end{array}$ & $\begin{array}{c}\text { Cohort 1 } \\
(\boldsymbol{n}=\mathbf{8})\end{array}$ & $\begin{array}{c}\text { Cohort 2 } \\
(\boldsymbol{n}=\mathbf{3 0})\end{array}$ \\
\cline { 2 - 5 } & $\boldsymbol{n}(\mathbf{\%})$ & $\boldsymbol{n}(\boldsymbol{\%})$ & $\boldsymbol{n}(\boldsymbol{\%})$ & $\boldsymbol{n}(\boldsymbol{\%})$ \\
\hline Primary care (FM, IM, Peds) & $4(44.4)$ & $18(62.1)$ & $4(50.5)$ & $19(63.3 \%)$ \\
\hline $\begin{array}{l}\text { Specialty care (e.g., cardiology, } \\
\text { emergency medicine, } \\
\text { anesthesiology) }\end{array}$ & $2(22.2)$ & $6(20.7)$ & $2(25.0)$ & $6(20.2)$ \\
\hline $\begin{array}{l}\text { Nonclinical (e.g., research, public } \\
\text { health, anthropology, nutrition } \\
\text { science) }\end{array}$ & $3(33.3)$ & $5(17.2)$ & $2(25.5)$ & $5(16.7)$ \\
\hline
\end{tabular}

Note. $\mathrm{FM}=$ family medicine; $\mathrm{IM}=$ internal medicine; Peds = pediatrics.

Only two faculty members changed rank during the year following the session, with one being promoted from instructor to assistant professor and another promoted from assistant to associate professor (Table 3). Cohort 2 faculty reported that their primary roles changed in terms of teaching activities involved in medical student education, while faculty in Cohort 1 reported no changes in teaching activities on the follow-up survey. Some changes were noted in tribal community engagement, especially among participants in Cohort 1, and the same was the case for diversity activities participants reported (Table 3). More detailed results for diversity activities are shown in Table 4.

Table 3

Faculty Rank, Teaching, Tribal Engagement, and Diversity Activities at 1-Year Follow-up

\begin{tabular}{|c|c|c|c|c|}
\hline \multirow{3}{*}{ Survey question } & \multicolumn{2}{|c|}{$\begin{array}{c}\text { Cohort } 1 \\
n=8\end{array}$} & \multicolumn{2}{|c|}{$\begin{array}{c}\text { Cohort } 2 \\
n=30\end{array}$} \\
\hline & Yes & No & Yes & No \\
\hline & $n(\%)$ & $n(\%)$ & $n(\%)$ & $n(\%)$ \\
\hline Change in rank & $1(12.5)^{\mathrm{a}}$ & $7(87.5)$ & $1(3.3)^{b}$ & $29(96.7)$ \\
\hline \multicolumn{5}{|l|}{$\begin{array}{l}\text { What teaching activities are you doing } \\
\text { now that you were not doing } \\
\text { previously? }\end{array}$} \\
\hline $\begin{array}{l}\text { Medical student education (including } \\
\text { precepting) }\end{array}$ & 0 & 0 & $5(16.7)$ & $2(6.7)$ \\
\hline $\begin{array}{l}\text { Resident education (including } \\
\text { precepting) }\end{array}$ & 0 & 0 & $1(3.3)$ & $6(20.0)$ \\
\hline $\begin{array}{l}\text { Physician Assistant education } \\
\text { (including precepting) }\end{array}$ & 0 & 0 & $1(3.3)$ & $6(20.0)$ \\
\hline $\begin{array}{l}\text { Education of other health care } \\
\text { professionals }\end{array}$ & 0 & 0 & $2(6.7)^{\mathrm{c}}$ & $5(16.7)$ \\
\hline
\end{tabular}


Table 3 con't

\begin{tabular}{|c|c|c|c|c|}
\hline \multirow{3}{*}{ Survey question } & \multicolumn{2}{|c|}{$\begin{array}{c}\text { Cohort } 1 \\
n=8\end{array}$} & \multicolumn{2}{|c|}{$\begin{array}{c}\text { Cohort } 2 \\
n=30\end{array}$} \\
\hline & Yes & No & Yes & No \\
\hline & $n(\%)$ & $n(\%)$ & $n(\%)$ & $n(\%)$ \\
\hline \multicolumn{5}{|l|}{$\begin{array}{l}\text { Which tribal community engagement } \\
\text { activities have changed? }\end{array}$} \\
\hline $\begin{array}{l}\text { Attend organization meetings or } \\
\text { conferences }\end{array}$ & $2(25.0)$ & $3(37.5)$ & $5(16.7)$ & $1(3.3)$ \\
\hline Participate in organization committees & $1(12.5)$ & $4(50.0)$ & $3(10.0)$ & $3(10.0)$ \\
\hline Leadership role & 0 & $5(62.5)$ & $5(16.7)$ & $1(3.3)$ \\
\hline Other activities & $2(25.0)^{d}$ & $3(37.5)$ & $2(6.7)^{\mathrm{e}}$ & $4(13.3)$ \\
\hline \multicolumn{5}{|l|}{$\begin{array}{l}\text { In the past year, have you been involved } \\
\text { with any of the following? (diversity } \\
\text { activities) }\end{array}$} \\
\hline $\begin{array}{l}\text { Working with Diversity Office on } \\
\text { student issues }\end{array}$ & $2(25.0)$ & $6(75.0)$ & $8(26.7)$ & $22(73.3)$ \\
\hline $\begin{array}{l}\text { Provided input regarding diversity on a } \\
\text { new faculty hire }\end{array}$ & $4(50.0)$ & $4(50.0)$ & $9(30.0)$ & $21(70.0)$ \\
\hline $\begin{array}{l}\text { Worked to retain a faculty member who } \\
\text { was a member of an underrepresented } \\
\text { minority group }\end{array}$ & $2(25.0)$ & $6(75.0)$ & $8(26.7)$ & $22(73.3)$ \\
\hline Other (see Table 4) & $7(87.5)$ & $1(12.5)$ & $8(26.7)$ & $22(73.3)$ \\
\hline
\end{tabular}

${ }^{\mathrm{a}}$ From instructor to assistant professor. ${ }^{\mathrm{b}}$ From assistant to associate professor. ${ }^{\mathrm{c}}$ Master's and $\mathrm{PhD}$ students (one course); post-baccalaureate students. ${ }^{\mathrm{d}}$ Other activities: developing research collaborations; practice at my tribal clinic. ${ }^{\mathrm{e}}$ Tribal outreach, visits, participation in community events; consulting with Native American research project (community engagement).

\section{Table 4}

\section{Involvement in Other Diversity Activities for Cohorts 1 and 2 at 1-Year Follow-up}

\section{Cohort 1}

Serve as faculty adviser on House Officer's Diversity Committee; serve as faculty member of Pediatric Diversity Committee. Asked to meet with a student who was feeling microaggressions.

Diversity within residency program; on department diversity committee.

Retention of AI [American Indian] students and residents in trouble; assisting with leadership transition of student faculty support group.

Curriculum development to add culturally appropriate information for a middle school program. Cultural activities for programs which include ceremony, workshops, and mentorship.

I direct the Indian Health Pathway through the Center of Health Equity, Diversity \& Inclusion at my university. I participate in pipeline programs, and actively recruit Native students to our medical school program.

Worked with department to increase diversity in residency recruitment.

Taught in Wy'east program. Attended some Cherokee events in Portland. 


\section{Table 4 con't}

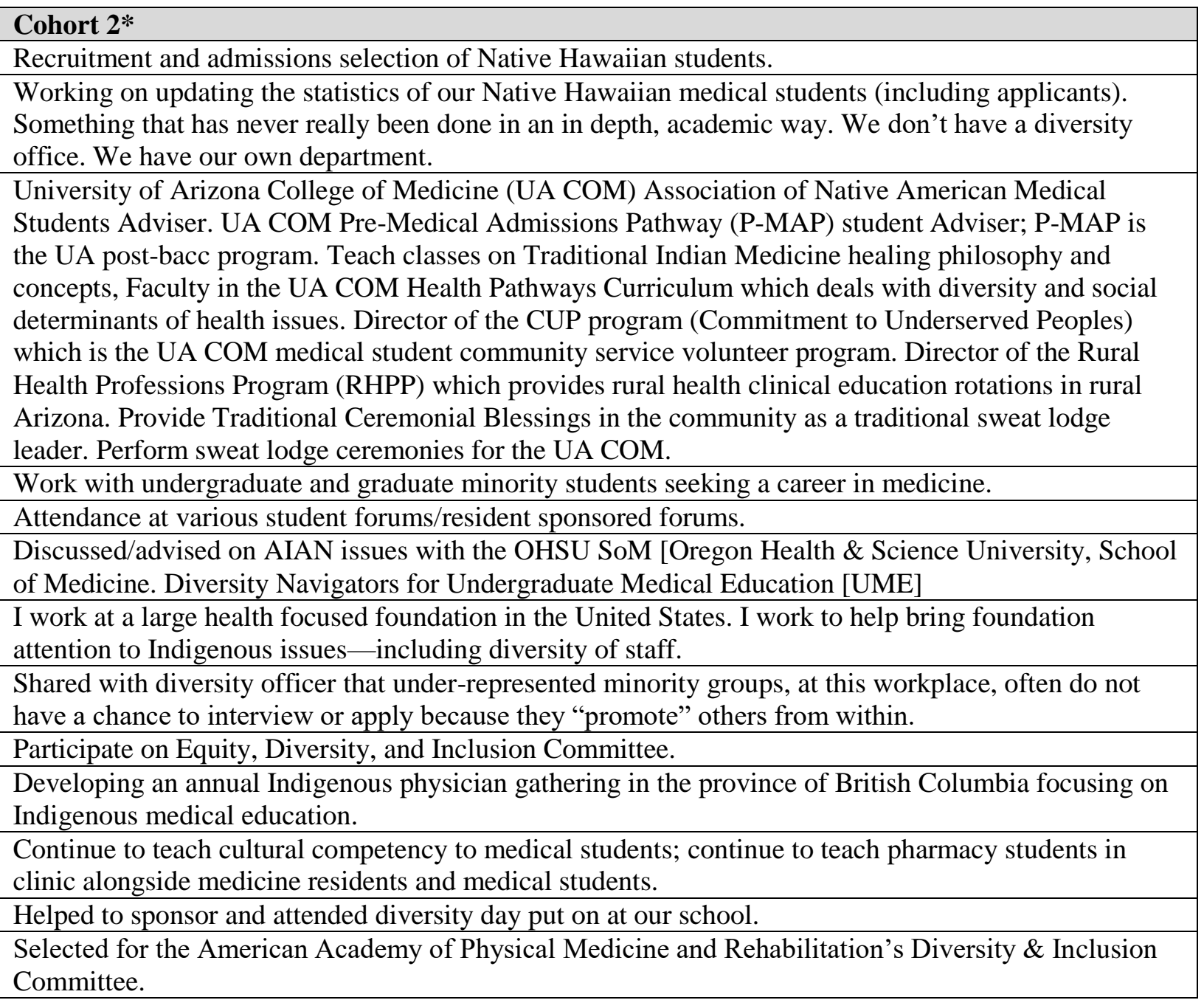

*Respondents were able to include more than one response.

Table 5 shows the DES scores for Cohort 1 compared to Cohort 2 at the time of the postsession survey and the 1-year follow-up survey. Only one statistically significant difference was noted. The Respect factor was higher in Cohort 1 compared to Cohort 2 at the post-session survey (Cohort 1: $M=3.96, S D=0.35$; Cohort 2: $M=3.42 ; S D=0.77 ; p=.01$ ).

Because of the small sample size in Cohort 1 and the lack of other differences, we combined Cohort 1 and 2 data for the comparison of post-session DES scores to the 1-year follow-up scores, which are shown in Table 6. Though the scores for Common Purpose, Equitable Reward and Recognition, Cultural Competence, Trust, a Sense of Belonging, and Appreciation of Individual Attributes increased slightly from post-session to 1 year, the changes were not statistically significant. The greatest change was for Respect, which increased from post-session to 1-year follow-up and achieved a $p$ value of .05 (Table 6). Access to Opportunity actually decreased between the two time periods, though this change was not statistically significant. 


\section{Table 5}

Diversity and Engagement Comparisons Between Cohort 1 and Cohort 2

\begin{tabular}{|c|c|c|c|c|c|c|c|c|c|c|}
\hline \multirow{3}{*}{$\begin{array}{c}\text { Diversity } \\
\text { and } \\
\text { Engagement } \\
\text { Survey } \\
\text { factor }\end{array}$} & \multicolumn{5}{|c|}{ Post-session } & \multicolumn{5}{|c|}{ 1-Year follow-up } \\
\hline & $\begin{array}{c}\text { Cohort } 1 \\
(n=9)\end{array}$ & $\begin{array}{c}\text { Cohort } 2 \\
(n=27)\end{array}$ & $\begin{array}{l}\text { Combined } \\
\text { Cohorts } \\
(n=36)\end{array}$ & \multirow[t]{2}{*}{$\boldsymbol{F}$} & \multirow{2}{*}{$\begin{array}{c}p \\
\text { value }\end{array}$} & $\begin{array}{c}\text { Cohort } 1 \\
\quad(n=8)\end{array}$ & $\begin{array}{c}\text { Cohort } 2 \\
(n=28)\end{array}$ & $\begin{array}{c}\text { Combined } \\
\text { Cohorts } \\
(n=36)\end{array}$ & \multirow[t]{2}{*}{$\boldsymbol{F}$} & \multirow[t]{2}{*}{$\begin{array}{c}p \\
\text { value }\end{array}$} \\
\hline & $\begin{array}{c}\text { Mean } \\
(S D)\end{array}$ & $\begin{array}{c}\text { Mean } \\
(S D)\end{array}$ & Mean $(S D)$ & & & $\begin{array}{c}\text { Mean } \\
(S D)\end{array}$ & $\begin{array}{c}\text { Mean } \\
(S D)\end{array}$ & $\operatorname{Mean}(S D)$ & & \\
\hline $\begin{array}{l}\text { Common } \\
\text { Purpose }\end{array}$ & $\begin{array}{l}3.89 \\
(0.60)\end{array}$ & $\begin{array}{c}3.62 \\
(0.85)\end{array}$ & $\begin{array}{l}3.69 \\
(0.79)\end{array}$ & 0.71 & .41 & $\begin{array}{l}4.19 \\
(0.53)\end{array}$ & $\begin{array}{l}3.80 \\
(0.75)\end{array}$ & $\begin{array}{l}3.89 \\
(0.72)\end{array}$ & 1.82 & .19 \\
\hline $\begin{array}{l}\text { Access to } \\
\text { Opportunity }\end{array}$ & $\begin{array}{c}4.33 \\
(0.71)\end{array}$ & $\begin{array}{c}4.04 \\
(0.82)\end{array}$ & $\begin{array}{l}4.11 \\
(0.79)\end{array}$ & 0.94 & .34 & $\begin{array}{l}4.06 \\
(0.62)\end{array}$ & $\begin{array}{l}3.75 \\
(1.03)\end{array}$ & $\begin{array}{c}3.82 \\
(0.96)\end{array}$ & 0.66 & .42 \\
\hline $\begin{array}{l}\text { Equitable } \\
\text { Reward and } \\
\text { Recognition }\end{array}$ & $\begin{array}{l}3.06 \\
(0.92)\end{array}$ & $\begin{array}{c}3.37 \\
(0.89)\end{array}$ & $\begin{array}{c}3.29 \\
(0.90)\end{array}$ & 0.83 & .37 & $\begin{array}{c}3.13 \\
(0.83)\end{array}$ & $\begin{array}{c}3.36 \\
(0.91)\end{array}$ & $\begin{array}{c}3.31 \\
(0.89)\end{array}$ & 0.42 & .52 \\
\hline $\begin{array}{l}\text { Cultural } \\
\text { Competence }\end{array}$ & $\begin{array}{c}3.61 \\
(0.22)\end{array}$ & $\begin{array}{c}3.43 \\
(0.76)\end{array}$ & $\begin{array}{c}3.48 \\
(0.67)\end{array}$ & 0.46 & .50 & $\begin{array}{c}3.72 \\
(0.49)\end{array}$ & $\begin{array}{c}3.63 \\
(0.82)\end{array}$ & $\begin{array}{c}3.65 \\
(0.75)\end{array}$ & 0.10 & .76 \\
\hline Trust & $\begin{array}{c}3.22 \\
(0.60)\end{array}$ & $\begin{array}{c}3.01 \\
(0.83)\end{array}$ & $\begin{array}{c}3.06 \\
(0.78)\end{array}$ & 0.49 & .49 & $\begin{array}{c}3.58 \\
(0.66)\end{array}$ & $\begin{array}{c}3.21 \\
(1.04)\end{array}$ & $\begin{array}{c}3.30 \\
(0.97)\end{array}$ & 0.89 & .35 \\
\hline $\begin{array}{l}\text { Sense of } \\
\text { Belonging }\end{array}$ & $\begin{array}{c}4.07 \\
(0.52) \\
\end{array}$ & $\begin{array}{c}3.96 \\
(0.67) \\
\end{array}$ & $\begin{array}{c}3.99 \\
(0.63) \\
\end{array}$ & 0.21 & .65 & $\begin{array}{c}4.00 \\
(0.44)\end{array}$ & $\begin{array}{c}4.00 \\
(0.84)\end{array}$ & $\begin{array}{c}4.00 \\
(0.76)\end{array}$ & 0.00 & 1.00 \\
\hline $\begin{array}{l}\text { Appreciation } \\
\text { of Individual } \\
\text { Attributes }\end{array}$ & $\begin{array}{c}4.04 \\
(0.51)\end{array}$ & $\begin{array}{c}3.61 \\
(0.62)\end{array}$ & $\begin{array}{c}3.71 \\
(0.62)\end{array}$ & 3.51 & .07 & $\begin{array}{c}4.00 \\
(0.47)\end{array}$ & $\begin{array}{c}3.76 \\
(0.82)\end{array}$ & $\begin{array}{c}3.81 \\
(0.75)\end{array}$ & 0.61 & .44 \\
\hline Respect & $\begin{array}{c}3.96 \\
(0.35) \\
\end{array}$ & $\begin{array}{c}3.25 \\
(0.79) \\
\end{array}$ & $\begin{array}{c}3.42 \\
(0.77) \\
\end{array}$ & 6.72 & .01 & $\begin{array}{c}3.88 \\
(0.56) \\
\end{array}$ & $\begin{array}{c}3.73 \\
(0.70)\end{array}$ & $\begin{array}{c}3.76 \\
(0.67) \\
\end{array}$ & 0.30 & .59 \\
\hline
\end{tabular}

\section{Table 6}

Diversity Engagement Post-Session and at 1-Year Follow-Up $(\mathrm{n}=36)$

\begin{tabular}{|l|c|c|c|}
\hline \multirow{2}{*}{$\begin{array}{c}\text { Diversity and Engagement Survey } \\
\text { factor }\end{array}$} & Post-session & 1-Year follow-up & \multirow{2}{*}{$\boldsymbol{p}$ value $^{\text {a }}$} \\
\cline { 2 - 3 } & Mean $(\boldsymbol{S D})$ & Mean $(\boldsymbol{S D})$ & .28 \\
\hline Common Purpose & $3.69(0.80)$ & $3.89(0.72)$ & .16 \\
\hline Access to Opportunity & $4.11(0.79)$ & $3.82(0.96)$ & .95 \\
\hline Equitable Reward and Recognition & $3.29(0.90)$ & $3.31(0.89)$ & .32 \\
\hline Cultural Competence & $3.48(0.67)$ & $3.65(0.75)$ & .26 \\
\hline Trust & $3.06(0.78)$ & $3.30(0.97)$ & .96 \\
\hline Sense of Belonging & $3.99(0.63)$ & $4.00(0.76)$ & .53 \\
\hline Appreciation of Individual Attributes & $3.71(0.62)$ & $3.81(0.75)$ & .05 \\
\hline Respect & $3.42(0.77)$ & $3.76(0.67)$ & \\
\hline
\end{tabular}

${ }^{\mathrm{a}}$ Independent samples $t$ test.

Table 7 shows the responses to the focus group questions, which reveal that mentoring that includes the cultural perspectives of AIAN is lacking, as is respect for these faculty from the academic community. Storytelling about patients and family centeredness are vitally important 
supportive attributes that can help them succeed. Participants often mentioned the programs they delivered and the mentoring they provided to others, and there is an important feature related to coming together that provides the "glue" that sustains this work. Cultural aspects of this work provide both strengths and weaknesses - the strengths in storytelling and weaknesses in being ignored by the academic community as relevant when there is much to be shared and learned by them that would strengthen all. In terms of what faculty development skills are needed, many of these focused on facilitating change (Table 7).

\section{Table 7}

Qualitative Findings From the Post-Session Focus Group

\begin{tabular}{|l|l|}
\hline \multicolumn{1}{|c|}{ Focus group question } & \multicolumn{1}{c|}{ Responses* } \\
\hline $\begin{array}{l}\text { 1. How has your academic } \\
\text { career gone so far? }\end{array}$ & - Very new to this but ok so far. \\
- Slow progress. \\
- Feels overwhelming sometimes. \\
- Lack of advocates in the academic setting. \\
- Academic relationships are less personal compared to what I am \\
used to in my culture. \\
- Nature of existing academic mentoring is not what I am looking \\
for. \\
- Being asked to be part of boards/committees to bring Indigenous \\
voice, but then no real higher-level support for recognizing any of \\
that; I got recruited to another university but my publications are \\
lacking because I'm so busy. \\
- I had knowledge that is valuable for Indigenous and non- \\
Indigenous students. Most universities don't recognize or value \\
my other cultural knowledge.
\end{tabular}




\section{Table 7 con't}

\begin{tabular}{|c|c|}
\hline Focus group question & Responses* \\
\hline $\begin{array}{l}\text { 3. What are you most proud of } \\
\text { in terms of academic } \\
\text { accomplishments? }\end{array}$ & $\begin{array}{l}\text { - Proud of those in the room who finished. It's awesome and } \\
\text { amazing to be a part of it. } \\
\text { - We've been gifted with something and it's how you use those gifts } \\
\text { and respect the teaching and honoring. } \\
\text { - Most proud to help a trainee who's struggling and proud of them, } \\
\text { not myself. } \\
\text { - Delivering curriculum and faculty development programs. } \\
\text { - We got the medical school to actually do the Indigenous health } \\
\text { teaching, we're currently fighting for space for this curriculum. } \\
\text { The few Indigenous faculty there are stretched so thin so can't do a } \\
\text { lot of mentorship. } \\
\text { - Being able to establish several programs reaching really young } \\
\text { kids to get interested in all health fields, and also college, faculty } \\
\text { development, working on getting into general med school } \\
\text { curriculum to influence. }\end{array}$ \\
\hline $\begin{array}{l}\text { 4. What does academic success } \\
\text { look like? }\end{array}$ & $\begin{array}{l}\text { - It's getting to do the things I want, the ones I'm excited about. } \\
\text { - Your department might not see the value in what you do. }\end{array}$ \\
\hline $\begin{array}{l}\text { 5. How does your culture inform } \\
\text { your experience as an } \\
\text { academic faculty member? }\end{array}$ & $\begin{array}{l}\text { - I approach working with others differently. They are more } \\
\text { traditional and directive, so I don't approach it that way. } \\
\text { - I like to work out solutions together instead of telling them what to } \\
\text { do. }\end{array}$ \\
\hline $\begin{array}{l}\text { 6. How has your culture fostered } \\
\text { or hindered your success as a } \\
\text { professional? }\end{array}$ & $\begin{array}{l}\text { - It makes my career richer-the patient stories. } \\
\text { - You have to give up a part of yourself to write grants and get } \\
\text { R01s. } \\
\text { - Constantly having to translate is hard. There's non-verbal and } \\
\text { verbal and having to force myself into verbal realm is } \\
\text { uncomfortable. } \\
\text { - We have cultural obligations that aren't recognized by the } \\
\text { university. } \\
\text { - There's no recognition of us being in an unsafe teaching } \\
\text { environment with "learners" (loose word I'll use), and the need for } \\
\text { self-care and balance after that; makes it a struggle to stay healthy } \\
\text { when there's ignorant racist comments happening in academia. } \\
\text { - Culture comes first and my profession comes second; culture is my } \\
\text { community, I work on relationships/engagement; if I'm not } \\
\text { representing appropriately then I step away. } \\
\text { - I think my profession sometimes gets in the way of who I am as an } \\
\text { Indigenous person; I might get teary ... my son is First Nations } \\
\text { (I'm Metis) and I've been trying to think about how do I talk to a } \\
\text { five-year-old about who he is. You're always fighting these } \\
\text { political battles, how the government defines you, how do I } \\
\text { describe this to a five-year-old? } \\
\text { - I try to bring my cultural lens into everything, walking in two } \\
\text { worlds; this is so different from being in an institution. When I'm } \\
\text { at a university I have to check back in to who I am. Slowly I'm } \\
\text { figuring it out. }\end{array}$ \\
\hline
\end{tabular}




\begin{tabular}{|l|l|}
\hline 7. What types of professional \\
$\begin{array}{l}\text { development help do you } \\
\text { need? }\end{array}$ & $\begin{array}{l}\text { - Mentorship and leadership skills development. } \\
\text { - How to run a staff. }\end{array}$ \\
& - Managing conflict, negotiation skills, how to help others. Nothing \\
& will change if you don't have the means to make the change, and \\
& knowing how to fight and not just take it. \\
& - Small group formats that include self-care activities. \\
\hline
\end{tabular}

*Minor edits to responses for clarity

\section{Limitations}

The limitations of this study include a lower than desired response rate to our core surveys and the lack of more extensive interactions with participants over time that could have provided meaningful data. Also, we neglected to include a question about peer support on the follow-up survey. We have since added this, and it will provide important information in future research. Importantly, very little is published on intervention research for Indigenous academic faculty as well as their learners. Yet another weakness is the small sample size that limits intervention trials that have more discriminatory power to determine what effectively supports and measurably improves the experience and advancement of Indigenous faculty. The strengths of this study include the success in recruiting diverse Indigenous participants and attaining a deep dive into their perspectives from both a quantitative and qualitative point of view.

\section{Discussion}

This study is among very few to assess a program specifically designed to support Indigenous faculty at U.S. medical schools. In 2006, Acosta and Olsen described a 1-year faculty development fellowship for AIAN physicians to prepare them for teaching and research careers in academic institutions, as well as a faculty development seminar series to help develop skills in teaching administration, practice management, research, and traditional healing. Unfortunately, these authors do not report on outcomes associated with their faculty development program. Buchwald and Dick (2011) used social network analysis to evaluate their Native Investigator Development Program, which was a career development program for junior AIAN researchers and represented a collaboration between the University of Washington and the University of Colorado, Denver. Their study included 29 trainees and assessed their scholarly productivity between 1998 and 2017, revealing that 106 manuscripts and 83 grant applications were produced (Buchwald \& Dick, 2011). The majority of this work was guided by three highly connected individuals, though both core faculty and program graduates were deeply involved in the collaborations.

Our experience in the pilot year of the IFF program revealed high participation and response rates. Institutions should increase their commitment to support these unique faculty and highlight their achievements in a way that underscores the importance of culture in medicine while also recognizing their humility. Many challenges exist similar to those reported in other studies (Rodriguez et al., 2015; Sánchez, et al., 2016). Our attempt to address these challenges served to further highlight their existence. Mentorship by other Indigenous faculty members is 
much desired but lacking. Time is spent by Indigenous faculty trying to serve and educate the academic community about these issues, which appears not to be recognized or appreciated. The sentiment among AIAN faculty is that system-wide trainings are done to "check a box" related to diversity rather than making meaningful change in the culture of inclusivity in an institution. Of note is that the commitment to Indigenous culture rests deep in the makeup of the participants in this project. National organizations that support AIAN physicians certainly exist (e.g., Association of American Indian Physicians, https://www.aaip.org), but the extent to which their programs are evaluated with longitudinal follow-up is unclear.

There is much to be learned about creating vital successful programs, both in terms of program-specific changes as well as assessment of these programs. We learned that a 1-day session to provide skills to Indigenous faculty does not provide either the rigor or depth necessary to sustain them over time. More mentoring and support by higher-level faculty and administration are needed, as well as the development of a cadre of Indigenous faculty to guide these efforts. Culture is vitally important to recognize and all can benefit from storytelling, which is so important to the compassion for patient care and regard for family that we found in this study. Non-Indigenous faculty could benefit from learning about Indigenous faculty culture, aspirations, and approaches to patient care. Much more respect and consideration are needed from non-Indigenous faculty and staff, who must see through a different lens when collaborating on education, clinical care, and research. Many of the institutes of the National Institutes of Health are now focusing health research on Indigenous people, especially to reduce health disparities and chronic illness (National Institute of Diabetes and Digestive and Kidney Diseases, 2020; Office of Behavioral and Social Science Research, 2020). These efforts should include AIAN physicians.

In terms of educational enhancement to the program, more supportive activities are needed. Matching each Indigenous faculty member with a coach providing additional tailored support may have been helpful. This coaching approach is now being used in recognition of culturally diverse students (Najibi et al., 2019), and using the same approach with faculty may be beneficial, though this deserves further study. Certainly, more support for faculty collaboration is also needed, but providing this support will depend on developing and retaining Indigenous faculty in the U.S. and elsewhere. Fostering the cohort effects, which results in meaningful connectedness in a year-round "learning community," is in its infancy, with the goal of fostering increased communication and collaboration on scholarly activities among participants. We learned that Indigenous faculty prefer small group interactions that include meaningful cultural activities as well as self-care. Given Indigenous faculty desire and passion to work with learners, it may benefit faculty development interventions to routinely include learners as part of a supportive intervention. Again, much more research is needed in these areas.

From a measurement perspective, we found not much had changed in a 1-year interval, which further suggests that more than a 1-day session is needed for impactful change. We had hoped that the IFF sessions would promote the development of supportive networks, but it is unclear whether this occurred, even though it was greatly desired. Competing demands of 
community service, education, and clinical care are not new to medical school faculty. However, additional challenges appeared to be predominant among Indigenous faculty, such as lack of culturally congruent mentorship, recognition from leadership, and support for efforts to improve tribal health and welfare. Despite these findings, the desire and passion to support tribal communities, families, and learners was prevalent. Much better measures are needed to capture these comprehensive issues than we had anticipated, and we are working on developing these now.

The Northwest Native American Center of Excellence aims to create a learning community, which will foster the success and advancement of Indigenous faculty at their respective institutions. In doing so, we aim to foster greater connection and skill building among the cohort(s), while better preparing participants to be successful within the milieu of their home institutions. The IFF in no way exempts the faculty's home institutions from the responsibility to support and develop all of their faculty; rather, the Forum exists to augment opportunities and support mechanisms at their home institutions. Operating in this way is intended to make extremely efficient use of resources across institutions and better understand the programming and measurement instruments needed to develop and operate similar programs.

\section{Conclusion}

In conclusion, we succeeded in implementing a program that addressed many of the challenges that Indigenous faculty face; however, we know more work is needed to understand supplemental efforts required over time and measures that allow more tailoring to participants' reactions to optimize such programs.

\section{References}

Acosta, D., \& Olsen, P. (2006). Meeting the needs of regional minority groups: The University of Washington's programs to increase the American Indian and Alaskan Native physician workforce. Academic Medicine, 81(10), 863-870. https://doi.org/10.1097/01.ACM.0000238047.48977.05

Association of American Medical Colleges. (2017). Applicant and Matriculant Data Tables: https://www.aamc.org/system/files/d/1/5-

2017_applicant_and_matriculant_data_tables.pdf.

Bowman, R. C. (2008). They really do go. Rural and Remote Health, 8(3), Article 1035. https://www.rrh.org.au/journal/article/1035

Brodt, E., Bruegl, A., Thayer, E. K., Eiff, M. P., Gonzales, K., Crespo, C., Spector, D., Kamaka, M., Carpenter, D.-A., \& Carney, P. A. (2018). Concerning trends in allopathic medical school faculty rank for Indigenous people: 2014-2016. Medical Education Online, 23(1). https://www.tandfonline.com/doi/full/10.1080/10872981.2018.1508267

Brodt, E., Empey, A., Mayinger, P., Fuqua Miller, M., Spector, D., Frutos, R., Bruegl, A., Zeisman-Pereyo, S., Olsen, P., \& Carney, P. A. (2019). Shifting the tide: Innovative strategies to develop an American Indian/Alaska Native physician workforce. Hawai $i$ Journal of Health \& Social Welfare, 78(12, Suppl. 3), 21-25. https://hawaiijournalhealth.org/past_issues/78.12.suppl3.htm 
Buchwald, D., \& Dick, R. W. (2011). Weaving the Native web: Using social network analysis to demonstrate the value of a minority career development program. Academic Medicine, 86(6), 778-786. https://doi.org/10.1097/ACM.0b013e318217e824

Cohen, J. J., Gabriel, B. A., \& Terrell, C. (2002). The case for diversity in the health care workforce. Health Affairs, 21(5), 90-102. https://doi.org/10.1377/hlthaff.21.5.90

Fang, D., Moy, E., Colburn, L., \& Hurley, J. (2000). Racial and ethnic disparities in faculty promotion in academic medicine. JAMA (Journal of the American Medical Association), 284(9), 1085-1092. https://doi.org/10.1001/jama.284.9.1085

Kunitz, S. J. (2008). Changing patterns of mortality among American Indians. American Journal of Public Health, 98(3), 404-411. https://doi.org/10.2105/AJPH.2007.114538

Marcinko, T. (2016, November 13). More Native American doctors needed to reduce health disparities in their communities. AAMC News. https://www.aamc.org/newsinsights/more-native-american-doctors-needed-reduce-health-disparities-theircommunities

Mayring, P. (2000). Qualitative content analysis. FQS (Forum: Qualitative Social Research), 1(2), Article 20. http://www.qualitativeresearch.net/index.php/fqs/article/view/1089/2385

Najibi S, Thayer EK, Deiorio N, Carney PA. Differences in Coaching Needs Among Underrepresented Minority Medical Students. Fam Med, 2019; 51(6):516-522. https://doi.org/10.22454/FamMed.2019.100305.

National Institute of Diabetes and Digestive and Kidney Diseases. (2020). Addressing health disparities in NIDDK diseases [Funding opportunity announcement]. https:/grants.nih.gov/grants/guide/pa-files/PA-18-412.html

Northwest Portland Area Indian Health Board. American Indian \& Alaska Native Community Health Profile - Idaho. Portland, OR; Northwest Tribal Epidemiology Center, 2014.

http://www.npaihb.org/images/epicenter_docs/IDEA/2014/IdReports/0_Idaho_Intro\&Contents.p df

Office of Behavioral and Social Science Research. (2020). https://obssr.od.nih.gov

Page, K. R., Castillo-Page, L., \& Wright, S. M. (2011). Faculty diversity programs in U.S. medical schools and characteristics associated with higher faculty diversity. Academic Medicine, 86(10), 1221-1228. https://doi.org/10.1097/ACM.0b013e31822c066d

Person, S. D., Jordan, C. G., Allison, J. J., Fink Ogawa, L. M., Caseillo-Page, L., Conrad, S., Nivet, M. A., \& Plummer, D. L. (2015). Measuring diversity and inclusion in academic medicine: The Diversity Engagement Survey. Academic Medicine, 90(12), 1675-1683. https://doi.org/10.1097/ACM.0000000000000921

Peterson, N. B., Friedman, R. H., Ash, A. S., Franco, S., \& Carr, P. L. (2004). Faculty selfreported experience with racial and ethnic discrimination in academic medicine. Journal of General Internal Medicine, 19(3), 259-265. https://doi.org/10.1111/j.15251497.2004.20409.x 
Price, E. G., Gozu, A., Kern, D. E., Powe, N. R., Wand, G. S., Golden, S., \& Cooper, L. A. (2005). The role of cultural diversity climate in recruitment, promotion, and retention of faculty in academic medicine. Journal of General Internal Medicine, 20(7), 565-571. https://doi.org/10.1111/j.1525-1497.2005.0127.x

Rodriguez, J. E., Campbell, K. M., \& Pololi, L. H. (2015). Addressing disparities in academic medicine: What of the minority tax? BMC Medical Education, 15, Article 6. https://doi.org/10.1186/s12909-015-0290-9

Sánchez, J. P., Poll-Hunter, N., Stern, N., Garcia, A. N., \& Brewster, C. (2016). Balancing two cultures: American Indian/Alaska Native medical students' perceptions of academic medicine careers. Journal of Community Health, 41(4), 871-880. https://doi.org/10.1007/s10900-016-0166-x

U.S. Census Bureau. (2010). 2000 and 2010 Census Profile: Portland-Vancouver-Hillsboro MSA. Population Research Center, Portland State University. https://ondeck.pdx.edu/population-research/sites/g/files/znldhr3261/files/2020-07/PortVanc-Hills_MSA_Census2010.pdf 